[Chem. Pharm. Bull.

[35(1) $370-376(1987)]$

\title{
Interactions between Furosemide and Spironolactone in Normal Subjects
}

\author{
KATSUYOSHI UChINO, ${ }^{*},{ }^{a}$ SADAO IsOZAKI, ${ }^{a, 1 a)}$ NAOMI TANAKA,${ }^{b, 1 b}$ \\ YUKIYA SAITOH $^{a}$ and FUJIO NAKAGAWA ${ }^{a}$ \\ Hospital Pharmacy and The First Department of Medicine, ${ }^{b}$ \\ Faculty of Medicine, University of Tokyo, 3-1, Hongo \\ 7-chome, Bunkyo-ku, Tokyo 113, Japan
}

(Received July 23, 1986)

\begin{abstract}
Furosemide (FD) and spironolactone (SP) were co-administrated to human volunteers, and the pharmacokinetics and time course of diuresis were examined. The time courses of FD plasma concentration and diuresis after a single co-administration of FD plain tablet and SP were similar to those in the case of FD plain tablet. However, the plasma concentration of SP metabolites (determined as canrenone) during the $2-6 \mathrm{~h}$ period after a single co-administration of FD plain tablet and SP was about $0.05-0.15 \mu \mathrm{g} / \mathrm{ml}$ higher than that after administration of SP. The steadystate distribution volume of SP metabolites after co-administration of FD plain tablet and SP was about $30 \%$ less than that after administration of SP, while the elimination rate constant of SP metabolites was about 1.5 -fold greater. The diuresis during $24 \mathrm{~h}$ after a single administration of SP was $0.1-0.31$.

After multiple administration of SP, the plasma concentration of SP metabolites during the $2-6 \mathrm{~h}$ period after co-administration of FD plain tablet and SP was also about $0.1-0.3 \mu \mathrm{g} / \mathrm{ml}$ higher than expected from the time course of SP metabolites simulated by using the data obtained after a single administration of SP. The elevation of SP metabolites concentration in plasma was prevented without any change of diuresis over $24 \mathrm{~h}$ by using FD retard capsule instead of FD plain tablet.

We concluded that the phenomenon of elevation of SP metabolites concentration in plasma was caused by FD plain tablet, which induced strong diuresis and considerable loss of body fluids within a short time.
\end{abstract}

Keywords —-furosemide; spironolactone; fluorigenic spironolactone metabolite; drug interaction; plasma concentration; diuresis; normal subject

Furosemide (FD) has been widely used for the treatment of cirrhotic patients with ascites. When FD is administered to such patients, side effects such as hypokalemia, disturbance of other electrolytes and hepatic encephalopathy have been reported. ${ }^{2}{ }^{2}$ Therefore, the concomitant administration of FD with spironolactone (SP) is recommended for the prophylaxis of hypokalemia and hepatic encephalopathy or the treatment of hyperaldosteronism. ${ }^{3}$ While side effects, mainly gynecomastia, have also been reported during chronic therapy with $\mathrm{SP}^{4}{ }^{4}$ their appearance depends on the dose and duration of the drug administration. ${ }^{4 d, e)}$ Therefore, care is necessary when both drugs are concomitantly administered to cirrhotic patients with ascites. There are few reports concerning the pharmacokinetic interactions between FD and SP after concomitant administration of the drugs in man. Homeidia et al. ${ }^{5)}$ have reported that the pharmacokinetics of FD was not influenced by SP. Nevertheless, the report did not examine the influence of FD on the pharmacokinetics of SP, or on the diuresis.

The present paper describes the pharmacokinetics and time course of diuresis after concomitant administration of FD and SP to human volunteers. 


\section{Experimental}

Drugs and Reagents_-Authentic FD and canrenone used for all experiments were supplied by Hoechst Japan Ltd., Tokyo, Japan and Dainippon Pharmaceutical Co., Ltd., Osaka, Japan, respectively. Plain tablets (Lasix $\left.{ }^{\circledR}\right)$ and retard capsules (Eutensine ${ }^{\circledR}$ ) containing $40 \mathrm{mg}$ of FD used in this study were obtained from Hoechst Japan Ltd. Tablets (Aldactone ${ }^{\circledR}$ A) containing $25 \mathrm{mg}$ of SP used in this study were obtained from Dainippon Pharmaceutical Co., Ltd. All the other solvents and reagents used were of reagent grade and were obtained from Wako Pure Chemical Industries Ltd., Tokyo.

Subjects - The subjects in this study were 3 males, $24-35$ years old, weighing $56-68 \mathrm{~kg}$. They were healthy as judged by ordinary clinical examinations and laboratory tests, and participated in this study after being informed of its purpose, procedure and potential hazards. The normal subjects were fed identical meals as reported previously ${ }^{6}$ and drank $2000 \mathrm{ml}$ of water a day dispensed in $500 \mathrm{ml}$ aliquots for each time period, i.e. 9:00-13:00, 13:00-17:00, 17:00-21:00 and $21: 00-9: 00$ on the next day. The control urine was collected at 9:00-21:00 and 21:00-9:00 on the next day for $5 \mathrm{~d}$ without drug in the same manner as described above. The urine volumes measured in each time-interval for $5 \mathrm{~d}$ were averaged to obtain control values. The diuresis after administration of each drug was evaluated by subtracting the mean control value from the urine volume observed after administration of the drug.

Single Dose Study_ FD Plain Tablet: The plasma and urine data after oral administration of FD plain tablet $(40 \mathrm{mg})$ are described in our preliminary report. ${ }^{6}$ )

SP: Each subject was given orally $50 \mathrm{mg}$ of SP as two $25 \mathrm{mg}$ tablets at 9:00 and blood samples were collected at $0.5,1,1.5,2,3,4,6,8,10$ and $24 \mathrm{~h}$ thereafter, while urine samples were collected at $0-6,6-12$ and $12-24 \mathrm{~h}$.

Concomitant Administration-FD Plain Tablet and SP (FD Plain Tablet + SP): Forty milligrams of FD plain tablet and $50 \mathrm{mg}$ of SP were concomitantly administered to three normal subjects at 9:00. Blood samples were collected at $0.5,0.75,1,1.5,2,2.5,3,4,5,6,8,10,12$ and $24 \mathrm{~h}$, and urine samples were collected at $1-2,2-3,3-4$, $4-5,5-6,6-8,8-10,10-12$ and $12-24 \mathrm{~h}$ after the administration of the two drugs.

FD Retard Capsule and SP (FD Retard Capsule + SP): Forty milligrams of FD retard capsule and $50 \mathrm{mg}$ of SP were concomitantly administered to one (K.U.) of three normal subjects at 9:00. The samples of blood and urine were collected in the same manner as described for the single dose study of FD plain tablet + SP.

Multiple Dose Study_ SP $(50 \mathrm{mg})$ was administered to three normal subjects once a day at 9:00 for $8 \mathrm{~d}$. On the 6th day, $50 \mathrm{mg}$ of SP plus $40 \mathrm{mg}$ of FD plain tablet were concomitantly administered. The blood samples on the $1 \mathrm{st}$ day after the drug administration were collected at $1,2,3,4$ and $24 \mathrm{~h}$, and urine samples were collected at $0-6,6-12$ and $12-24 \mathrm{~h}$. On the $2 \mathrm{nd}, 3 \mathrm{rd}, 4 \mathrm{th}, 5 \mathrm{th}$ and $7 \mathrm{th}$ days, blood samples were collected at 2 and $24 \mathrm{~h}$, and urine samples were collected in the same manner as described for the 1st day. The samples of plasma and urine on the 6th day were collected in the same manner as described for the single dose study of FD plain tablet + SP, and those on the 8th day were collected in the same manner as described for the single dose study of SP.

Collection and Treatment of Samples_- Blood was drawn through an indwelling cannula (Hakko ${ }^{\circledR}$ 9G, Hakko Co., Ltd., Tokyo) at the stated time in each study. Plasma was immediately separated from whole blood by centrifugation, and stored at $-20^{\circ} \mathrm{C}$ until the drug analysis. Urine was excreted just before drug administration and collected at the stated time-intervals thereafter in each study. Urine volume was measured and an aliquot was stored at $-20^{\circ} \mathrm{C}$ until analysis.

Determinations of FD and SP Metabolites in Plasma - The concentration of FD in plasma was analyzed by high-performance liquid chromatography (HPLC) according to the method of Uchino et al. ${ }^{7}$ )

When SP is administered to man, it is metabolized to canrenone, canrenoate, $7 \alpha$-thiomethylspirolactone, $6 \beta$ hydroxy- $7 \alpha$-thiomethylspirolactone and others. ${ }^{8)}$ Since many SP metabolites show fluorescence, total fluorigenic fraction of SP metabolites was used as a measure of effective concentration in this study. These metabolites in plasma were determined as equivalent concentration of canrenone by the modified method of Sadée et al. ${ }^{9 a}$ ) Accordingly, $2 \mathrm{ml}$ of $2 \mathrm{M}$ hydrochloric acid was added to $1 \mathrm{ml}$ of plasma. After standing for $15 \mathrm{~min}$ at room temperature, the mixture was extracted with $5 \mathrm{ml}$ of methylene chloride by shaking for $5 \mathrm{~min}$ on a mechanical shaker, followed by centrifugation on a KN-70 centrifuge (Kubota Seisakusho, Tokyo) at $1680 \mathrm{~g}$ for $5 \mathrm{~min}$. Then $4 \mathrm{ml}$ of the upper organic phase was transferred to another test tube, and $2 \mathrm{ml}$ of $0.1 \mathrm{M}$ sodium hydroxide was added. The mixture was rapidly stirred for $1 \mathrm{~min}$ with a Thermo-Mixer TM-105 (Thermonics Co., Ltd., Tokyo). After centrifugation, $3 \mathrm{ml}$ of the upper organic phase was transferred to another test tube, to which $4 \mathrm{ml}$ of $62 \%$ sulfuric acid (62 volumes of $96 \%$ sulfuric acid and 38 volumes of distilled water) was added. The mixture was shaken for 10 min and centrifuged for $5 \mathrm{~min}$. The upper organic phase was removed by aspiration, and the aqueous phase was allowed to stand for $1 \mathrm{~h}$ at room temperature. This solution was used to determine the concentration of SP metabolites in plasma. The apparatus used was a Hitachi 204 fluorescence spectrophotometer (Hitachi Seisakusho, Hitachi, Japan) and the excitation and emission wavelengths for fluorimetric detection of canrenone were set at 465 and $525 \mathrm{~nm}$, respectively. The calibration curve for canrenone concentration in plasma was linear over the concentration range of 0.025 to $0.4 \mu \mathrm{g} / \mathrm{ml}$ and passed through the origin.

Calculation of Pharmacokinetic Parameters from Time Course of Plasma Concentration of FD and SP Metabolites - The disposition of FD was approximated by a one-compartment open model with apparent first 
order absorption and elimination processes, and that of SP metabolites was approximated by two-compartment open model with apparent first order appearance, distribution and elimination processes. The pharmacokinetic parameters of FD or SP metabolites were calculated by using a nonlinear least-squares microcomputer program (MULTI). ${ }^{10}$ The disposition of SP metabolites was also calculated by using model-independent analysis. ${ }^{11)}$

\section{Results}

The time courses of plasma concentration of FD and diuresis after a single administration of FD plain tablet, or of FD plain tablet + SP in three normal subjects are shown in Fig. 1. The data on FD plain tablet in the upper and lower figures were taken from the previous report. ${ }^{6)}$ The time course of FD plasma concentration after administration of FD plain tablet + SP was similar to that in the case of FD plain tablet. The diuresis in both cases reached a maximum value of 1.51 within $3 \mathrm{~h}$, and decreased gradually to about 1.21 during $24 \mathrm{~h}$.

The plasma concentration of SP metabolites after a single administration of SP reached a maximum value of $0.3-0.45 \mu \mathrm{g} / \mathrm{ml}$ within $3 \mathrm{~h}$. However, the plasma concentration of SP metabolites during the $2-6 \mathrm{~h}$ period after a single administration of FD plain tablet $+\mathrm{SP}$ was 0.05 to $0.15 \mu \mathrm{g} / \mathrm{ml}$ higher than that after SP administration (Fig. 2). In all subjects, the rate constants of appearance $\left(k_{\mathrm{ap}}\right)$ of SP metabolites in the blood were comparable after administrations of SP and FD plain tablet + SP. The steady-state distribution volume $\left(V d_{\mathrm{ss}}\right)$ of SP metabolites after administration of FD plain tablet + SP decreased by about $30 \%$ compared to that after SP administration. The elimination rate constant $\left(k_{\mathrm{e} 1}\right)$ of SP metabolites after administration of FD plain tablet + SP was about 1.5 -fold higher than that after SP administration. However, total body clearance $(C l)$ of SP metabolites in each subject varied after administration of FD plain tablet + SP. In one subject (Y. Y.), $C l$ after SP

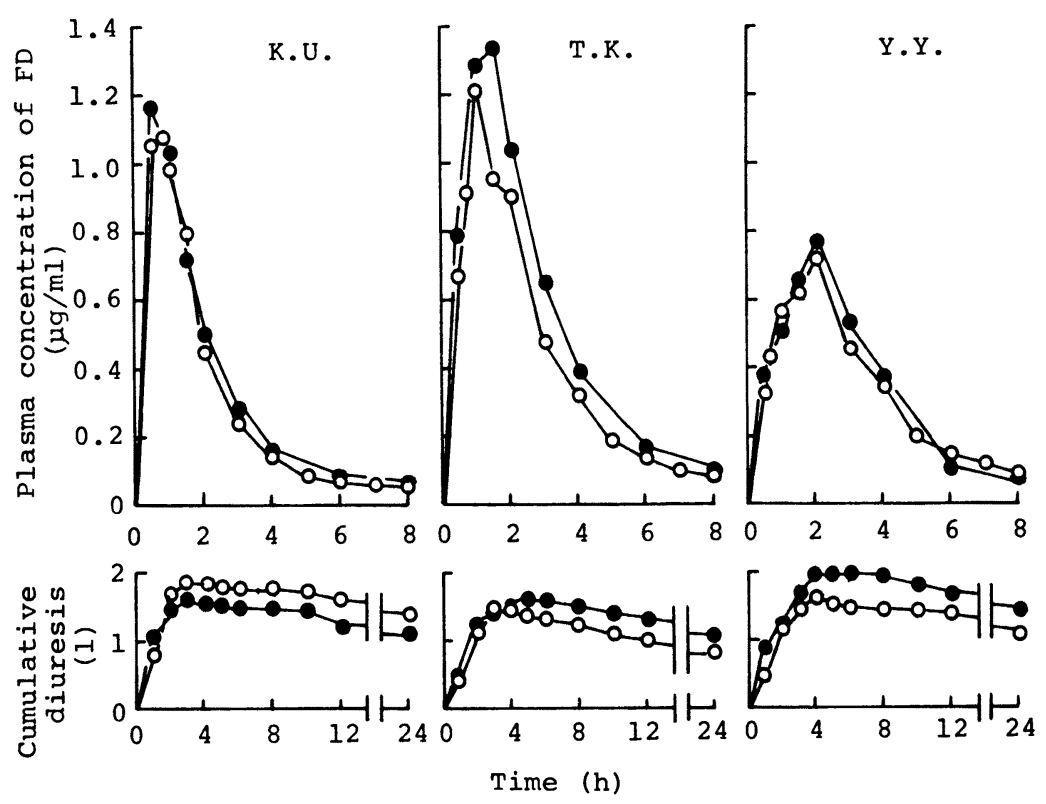

Fig. 1. Time Courses of Plasma Concentration of FD (Upper) and Cumulative Diuresis (Lower) in Three Normal Subjects after Administration of $40 \mathrm{mg}$ of FD Plain Tablet and Concomitant Administration of $40 \mathrm{mg}$ of FD Plain Tablet with $50 \mathrm{mg}$ of SP (FD Plain Tablet + SP)

Observed points for FD plain tablet in the upper and lower figures are taken from the previous study (ref. 6). $\bigcirc$, FD plain tablet; 9 , FD plain tablet + SP. 


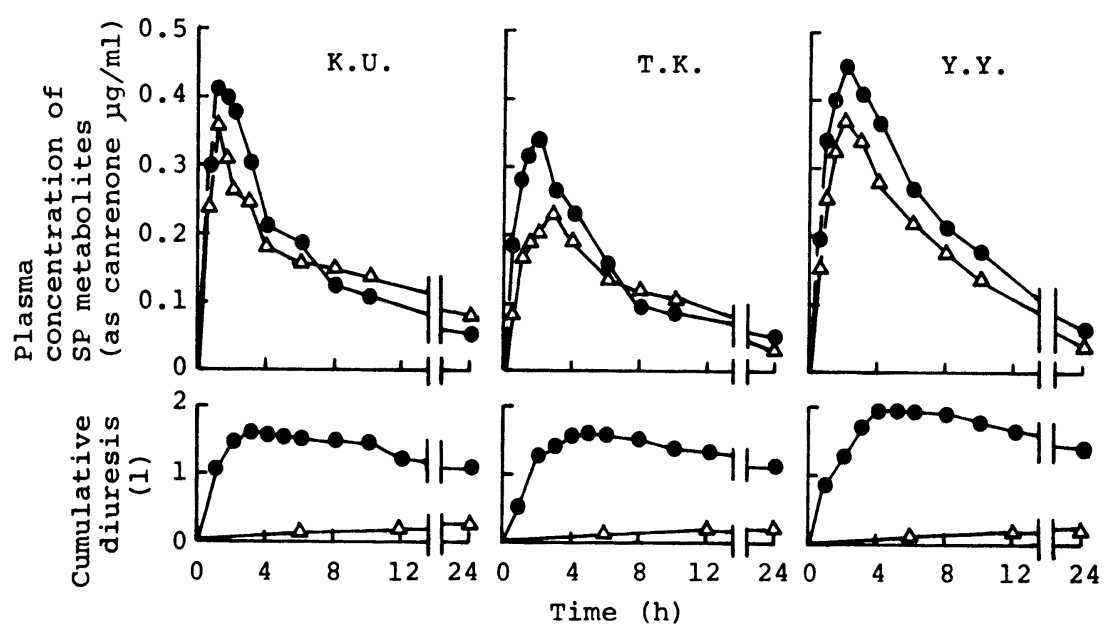

Fig. 2. Time Courses of Plasma Concentration of SP Metabolites (Upper) and Cumulative Diuresis (Lower) in Three Normal Subjects after Administration of $50 \mathrm{mg}$ of SP and Concomitant Administration of $40 \mathrm{mg}$ of FD Plain Tablet with $50 \mathrm{mg}$ of SP (FD Plain Tablet + SP)

Observed points for diuresis after FD plain tablet + SP are the same data as plotted in Fig. 1. $\triangle$, SP;, FD plain tablet $+\mathrm{SP}$.

TABLE I. Pharmacokinetic Parameters of SP Metabolites Obtained after Single Administration of SP or Concomitant Administration of FD Plain Tablet with SP (FD Plain Tablet + SP)

\begin{tabular}{|c|c|c|c|c|c|c|c|c|c|c|}
\hline \multirow{2}{*}{ Drug } & \multirow{2}{*}{$\begin{array}{l}\text { Dose } \\
(\mathrm{mg})\end{array}$} & \multirow{2}{*}{ Subjects } & \multicolumn{8}{|c|}{ Parameters } \\
\hline & & & $\begin{array}{c}\alpha \\
\left(h^{-1}\right)\end{array}$ & $\begin{array}{c}\beta \\
\left(h^{-1}\right)\end{array}$ & $\begin{array}{c}\left.k_{\mathrm{ap}}^{a}\right) \\
\left(\mathrm{h}^{-1}\right)\end{array}$ & $\begin{array}{c}k_{\mathrm{e} 1} \\
\left(\mathrm{~h}^{-1}\right)\end{array}$ & $\begin{array}{c}A U C \\
(\mu \mathrm{g} / \mathrm{ml} \cdot \mathrm{h})\end{array}$ & $\begin{array}{l}M R T \\
\text { (h) }\end{array}$ & $\begin{array}{c}V d_{\mathrm{ss}}^{b)} \\
(1)\end{array}$ & $\begin{array}{l}C l^{c)} \\
(\mathrm{l} / \mathrm{h})\end{array}$ \\
\hline \multirow[t]{6}{*}{ SP } & 50 & & & & & & & & & \\
\hline & & K.U. & 1.076 & 0.031 & 1.306 & 0.108 & 6.327 & 31.08 & 245.6 & 7.90 \\
\hline & & T.K. & 0.324 & 0.032 & 0.566 & 0.109 & 3.616 & 24.05 & 338.8 & 14.09 \\
\hline & & Y.Y. & 0.393 & 0.058 & 0.668 & 0.142 & 4.466 & 15.01 & 160.7 & 10.71 \\
\hline & & Mean \pm & 0.598 & 0.040 & 0.847 & 0.120 & 4.803 & 23.38 & 248.4 & 10.90 \\
\hline & & S.D. & 0.416 & 0.015 & 0.401 & 0.019 & 1.387 & 8.06 & 89.1 & 3.10 \\
\hline \multirow{9}{*}{$\begin{array}{l}\text { FD } \\
\text { Plain } \\
\text { tablet } \\
+ \text { SP }\end{array}$} & & & & & & & & & & \\
\hline & & & & & & & & & & \\
\hline & 40 & & & & & & & & & \\
\hline & 50 & & & & & & & & & \\
\hline & & K.U. & 0.798 & 0.064 & 1.223 & 0.201 & 3.878 & 13.73 & 177.0 & 12.89 \\
\hline & & T.K. & 0.057 & 0.056 & 0.742 & 0.208 & 3.189 & 14.34 & 224.8 & 15.67 \\
\hline & & Y.Y. & 0.362 & 0.079 & 0.728 & 0.154 & 4.675 & 11.30 & 120.9 & 10.71 \\
\hline & & Mean \pm & 0.406 & 0.066 & 0.898 & 0.188 & 3.914 & 13.12 & 174.2 & 13.06 \\
\hline & & S.D. & 0.372 & 0.012 & 0.282 & 0.029 & 0.744 & 1.61 & 52.0 & 2.49 \\
\hline
\end{tabular}

a) Rate constant of appearance of SP metabolites. b) Calculated as $V d_{\mathrm{ss}}=\operatorname{dose} \cdot f \cdot M R T / A U C, f=1 . \quad$ c) Calculated as $C l=$ $V d_{\mathrm{ss}} / M R T$.

administration was in fair agreement with that after administration of FD plain tablet + SP, whereas $\mathrm{Cl}$ after SP administration in the other subjects was larger than that after administration of FD plain tablet + SP. The mean residence time $(M R T)$ of SP metabolites 


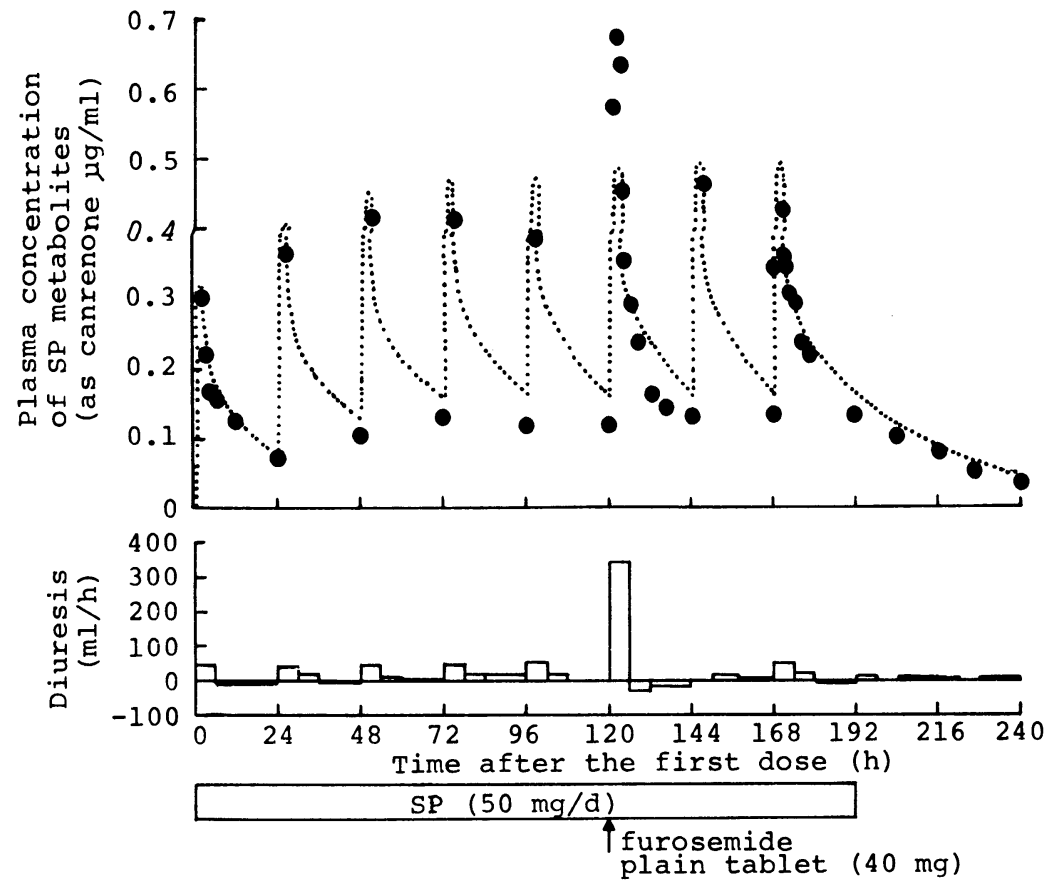

Fig. 3. Time Courses of Plasma Concentration of SP Metabolites (Upper) and Diuresis (Lower) in a Normal Subject (K.U.) during and after an Oral Multiple Administration of $50 \mathrm{mg}$ of SP Once a Day

The dotted line in the upper figure represents the simulation based on pharmacokinetic parameters obtained from the single-dose study of SP.

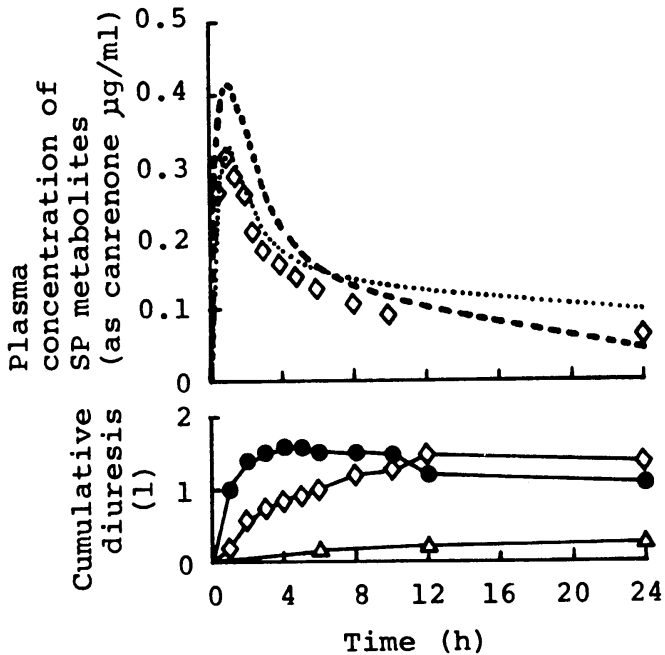

Fig. 4. Time Courses of Plasma Concentration of SP Metabolites (Upper) and Cumulative Diuresis (Lower) in a Normal Subject (K.U.) after Oral Concomitant Administration of 50 $\mathrm{mg}$ of SP with $40 \mathrm{mg}$ of FD Retard Capsule

In the upper figure, dotted and broken lines represent the simulations based on pharmacokinetic parameters obtained from single-dose studies of SP and $\mathrm{SP}+\mathrm{FD}$ plain tablet as shown in Figs. 1 and 2 , respectively. In the lower figure, data points $(\bullet, \triangle)$ for diuresis are taken from Figs. 1 and 2, respectively.

decreased by administration of FD plain tablet + SP (Table I). $V d_{\mathrm{ss}}$ and $C l$ of SP metabolites calculated by compartment analysis were approximately consistent with those obtained by model-independent analysis. The diuresis over $24 \mathrm{~h}$ after a single administration of SP was very weak, $0.1-0.31$.

A typical time course after multiple administration of $50 \mathrm{mg}$ of SP once a day in a normal 
subject (K. U.) is shown in Fig. 3. The plasma concentrations of SP metabolites observed every day fitted the time course after multiple administration of SP which was simulated by using the pharmacokinetic parameters of SP metabolites after a single administration of SP, with the exception of the 6th day. After a single administration of FD plain tablet + SP, the plasma concentration of SP metabolites during the $2-6 \mathrm{~h}$ period on the 6 th day was about 0.1 to $0.3 \mu \mathrm{g} / \mathrm{ml}$ higher than that on the 5 th day. On the 7 th day, the plasma concentration of SP metabolites reverted to the simulated curve.

The diuresis after multiple administration of SP was about 0.31 a day except on the 6 th and 7 th days. The diuresis profiles on these days were similar to that after a single administration of SP. On the 6th day, the diuresis was 1.21 a day and the profile was similar to that after a single administration of FD plain tablet + SP. The diuresis on the 7 th day was -0.61 a day (less than the control value) (Fig. 3).

To control the elevation of plasma concentration of SP metabolites produced by the concomitant administration of FD plain tablet $+\mathrm{SP}, 50 \mathrm{mg}$ of SP and $40 \mathrm{mg}$ of FD retard capsule were concomitantly administered to a subject (K.U.). The time course of plasma concentration of SP metabolites during the $2-6 \mathrm{~h}$ period was similar to that after SP administration (Fig. 4). $V d_{\mathrm{ss}}$ of SP metabolites after administration of FD retard capsule $+\mathrm{SP}$ was 249.61 , which was similar to that after SP administration, 245.6 l. The $k_{\mathrm{el}}$ and $C l$ of SP metabolites after administration of FD retard capsule $+\mathrm{SP}$ were $0.174 \mathrm{~h}^{-1}$ and $13.2 \mathrm{l} / \mathrm{h}$, respectively, while the corresponding values after SP administration were $0.108 \mathrm{~h}^{-1}$ and 7.9 1/h. However, the value for $M R T$ after administration of FD retard capsule $+\mathrm{SP}, 20.1 \mathrm{~h}$, was smaller than that after SP administration, $31.08 \mathrm{~h}$. The diuresis measured during a $24 \mathrm{~h}$ period after administrations of FD retard capsule + SP and FD plain tablet + SP amounted to 1.45 and 1.501 , respectively. However, quite different diuresis profiles were observed between the two dosage forms.

\section{Discussion}

The disposition of FD and time course of diuresis were not influenced by concomitant administration of SP. A similar finding with respect to intravenous administration of FD was reported by Homeidia et al. ${ }^{5)}$

It has been reported that SP after oral administration is metabolized into many metabolites. ${ }^{8)}$ Canrenone, one of these metabolites, was measured by fluorimetric assay in previous studies. ${ }^{9)}$ However, the method lacks specificity, as other fluorigenic metabolites were measured concomitantly. ${ }^{13)}$ Recently, SP and its metabolites in plasma after oral administration of the drug were determined by HPLC separation by Overdiek et al. ${ }^{8 d}$ ) However, the fluorimetric method seems to be the appropriate one for a study concerning SP therapy, since the assay value obtained by this method most probably provides a good measure of effective concentration. ${ }^{12)}$ The fraction of SP metabolites in plasma was determined as concentration of canrenone by the fluorimetric method in this study.

The time course of SP metabolites after multiple administration of $50 \mathrm{mg}$ of SP was linear in the present study. The pharmacokinetics of canrenone measured by HPLC after a single or multiple administration of SP has been reported to be non-linear. ${ }^{12 a, 13)}$ This discrepancy may be explained by the fact that the dose of SP giving in non-linear pharmacokinetics of canrenone in the other reports was 2- to 10- fold higher than that used in the present study, and furthermore the inter-dose interval in the other reports was shorter.

The elevation of plasma concentration of SP metabolites by administration of FD plain tablet + SP observed in the single- and multiple-dose studies may arise as follows. Since FD plain tablet induced strong diuresis, and considerable amount of body fluids were lost in normal subjects within a short time, $V d_{\mathrm{ss}}$ of SP metabolites might become smaller. Such a 
decrease of $V d_{\mathrm{ss}}$ would result in the elevation of SP metabolites concentration in plasma.

It was concluded that the alteration of $\mathrm{Cl}$ of SP metabolites by administration of FD plain tablet $+\mathrm{SP}$ arise from individual variations in the extents of decrease of $V d_{\mathrm{ss}}$ and $k_{\mathrm{el}}$.

To prevent the elevation of plasma concentration of SP metabolites, FD should be supplied at a rate such that gentle diuresis is maintained for a long time. We reported that the diuresis after administration of retard capsule as a sustained-release dosage form of FD was moderately prolonged compared to that after administration of FD plain tablet, and furthermore the diuresis during a $24 \mathrm{~h}$ period was the same for the two dosage forms. ${ }^{6}$ ) When FD retard capsule and SP were concomitantly administered to a normal subject, the plasma concentration during the $2-6 \mathrm{~h}$ period and $V d_{\mathrm{ss}}$ of SP metabolites after a single administration of FD retard capsule + SP were similar to those after SP administration, and the diuresis over $24 \mathrm{~h}$ in the case of FD retard capsule $+\mathrm{SP}$ was also similar to that after administration of FD plain tablet + SP (Fig. 4). It is suggested that the elevation of SP metabolites concentration in plasma observed after concomitant administration of SP and FD plain tablet is caused by the strong diuresis induced by FD plain tablet. Thus, we succeeded in preventing the elevation of SP metabolites concentration in plasma without affecting diuresis over $24 \mathrm{~h}$ by using FD retard capsule instead of FD plain tablet.

When the concomitant administration of FD with SP is required for the treatment of cirrhotic patients with ascites, the plasma concentration of SP metabolites will probably be elevated by the administration of FD plain tablet. Thus, the use of FD retard capsule should reduce the incidence of side effects following SP administration.

Acknowledgement This work was supported in part by a Grant-in-Aid for Co-operative Research (60304083) from The Ministry of Education, Science and Culture of Japan. The authors are grateful to Mrs. Michele McNeil for assistance in the preparation of this manuscript.

\section{References and Notes}

1) a) Present address: Hospital Pharmacy, Tokyo Teishin Hospital, 14-23, Fujimi 2-chome, Chiyoda-ku, Tokyo 102, Japan; b) Present address: The Department of Medicine, Institute of Clinical Medicine, University of Tsukuba, Sakura-mura, Nihari-gun, Ibaraki 305, Japan.

2) S. Sherlock, B. Senewiratne, A. Scott and J. G. Walker, Lancet, I, 1049 (1966); C. A. Naranjo, U. Busto and L. Cassis, Am. J. Hosp. Pharm., 35, 794 (1978); C. A. Naranjo, E. Pontigo, C. Valdengro, G. Gonzalez, I. Ruiz and U. Busto, Clin. Pharmacol. Ther., 25, 154 (1979).

3) AMA Division of Drug, "AMA Drug Evaluations," 5th ed., American Medical Association, Philadelphia, 1983, pp. 759-760; A. G. Gilman, L. S. Goodman, T. W. Rall and F. Murad, "The Pharmacological Basis of Therapeutics," 7th ed., MacMillan Publishing Co., New York, 1985, p. 901.

4) a) N. M. Mann, J. Am. Med. Assoc., 184, 778 (1963); b) R. M. Sussman, Lancet, I, 58 (1963); c) E. Clark, J. Am. Med. Assoc., 193, $163(1965) ;$ d) R. Caminos-Torres, L. Ma and P. J. Snyder, J. Clin. Endocrinol. Metab., 45, 255 (1977); e) G. Schrijver and M. H. Weinberger, Clin. Pharmacol. Ther., 25, 33 (1979).

5) M. Homeidia, C. Roberts and R. Branch, Clin. Pharmacol. Ther., 21, 402 (1977).

6) K. Uchino, S. Isozaki, J. Amano, N. Tanaka, Y. Saitoh, F. Nakagawa, Z. Tamura and H. Oka, J. PharmacobioDyn., 6, 684 (1983).

7) K. Uchino, S. Isozaki, Y. Saitoh, F. Nakagawa, Z. Tamura and N. Tanaka, J. Chromatogr., 308, 241 (1984).

8) a) A. Karim, Drug Metab. Rev., 8, 151 (1978); b) A. Karim, J. Hribar and M. Doherty, Xenobiotica, 7, 585 (1977); c) U. Abshagen, H. Rennekamp and G. Luszpinski, Naunyn Schmiedberg Arch. Pharmacol., 296, 37 $(1976) ; d)$ H. W. P. M. Overdiek, W. A. J. J. Hermens and F. W. H. M. Merkus, Clin. Pharm. Ther., 38, 469 (1985).

9) a) W. Sadée, M. Dagcioglu and S. Riegelman, J. Pharm. Sci., 61, $1126(1972) ;$ b) C. Gochman and C. L. Gant, J. Pharmacol. Exp. Ther., 135, 1312 (1962).

10) K. Yamaoka, Y. Tanigawara, T. Nakagawa and T. Uno, J. Pharmacobio-Dyn., 4, 879 (1981).

11) K. Yamaoka, T. Nakagawa and T. Uno, J. Pharmacokinet. Biopharm., 6, 547 (1978); L. Z. Benet and R. L. Galeazzi, J. Pharm. Sci., 68, 1071 (1979).

12) U. Abshager, E. Besenfelder, R. Endele, K. Kooch and B. Neubert, Eur. J. Clin. Pharmacol., 16, 255 (1979); W. Sadée and G. C. M. Beelen, "Drug Level Monitoring Analytical Technique, Metabolism, and Pharmaceuticals," John Wiley \& Sons, Inc., New York, 1980, pp. 423-426.

13) P. C. Ho, D. W. A. Bourne, E. J. Triggs and V. Healewood, Eur. J. Clin. Pharmacol., 27, 441 (1984). 